\title{
Selbstreflexive Verortung
}

\author{
Brigitte Kukovetz, Manfred Leonhardt, Rainer Loidl-Keil, Klaus Posch
}

\begin{abstract}
Sozialwirtschaftliche Einrichtungen positionieren und engagieren sich in und für gleichberechtigte Partnerschaften mit der Privatwirtschaft und der öffentlichen Hand. Eine klassische Frage stellt sich dabei nach wie vor: Was ist die Sozialwirtschaft eigentlich? Welche Unternehmen sind sozialwirtschaftlich, welche nicht? Mit dem »Sozialwirtschaft-Kompass « wird ein mehrdimensionales System geschaffen, das eine Verortung sozialwirtschaftlicher Organisationen erlaubt und dadurch eine Positionierung und Reflexion der eigenen Organisation ermöglicht.
\end{abstract}

Im herkömmlichen Verständnis, das der Tradition und Ideengeschichte der Markt-Staat-Dichotomie verhaftet ist, werden soziale Einrichtungen in der Abgrenzung von anderen Sektoren beschrieben: Sie wären gegenüber dem Staat »nicht öffentlich « und gegenüber dem Markt »nicht kommerziell «, und sie wären gegenüber dem informellen Sektor »nicht informell «.

Geht es um die Klärung der Entstehung moderner Organisationsformen und institutioneller Arrangements, so möchten wir eingetretene Pfade verlassen und einen alternativen Ansatz vorstellen. Die altbekannte Dichotomie »nicht profitorientiert « und »nicht privat « lässt wenig Raum für eine feinere Identitätsbildung und positiv bestimmte Profilierung. Selbst neuere Positionierungsversuche verlassen selten diese Perspektive. In diesem Feld existieren weitere Bezeichnungen mit Tradition, vor allem jene des »Dritten Sektors« und der »Non-Profit-Organisationen «. Gerade Letztere wird in einem traditionsreichen Begriffsverständnis vorrangig über das Fehlen einer Gewinnorientierung definiert. In einem weiteren Verständnis von Ressourcen und »Mehrwerten « kann ein solches allerdings nicht aufrecht erhalten bleiben.

Der Begriff »Sozialwirtschaft « spezifiziert sich erstens dahingehend, als darunter nicht alle Non-Profit- Organisationen oder all jene des »Dritten Sektors « zu verstehen sind; zweitens begreift er die Organisationen im Feld sozialer Dienstleistungen als wirtschaftliche Unternehmungen. Zwar stellt der Begriff »Sozialwirtschaft « also eine grobe inhaltliche Spezifizierung dar, bleibt aber noch vage, weshalb er von vielen Autorinnen und Autoren zu Recht kritisiert wird.

Beispielsweise zieht Joachim Merchel es vor, von »Einrichtungen der Sozialen Arbeit« zu sprechen, wobei er selbst keine stringente Definition dieser Einrichtungen vorweist. Er beschreibt die Einrichtungen über ihren Gegenstand, die sozialen Dienstleistungen. Soziale Dienstleistungen charakterisieren sich demnach in ihrer Immaterialität und Intangibilität, Unteilbarkeit und Nicht-Speicherbarkeit, Integration des externen Faktors,

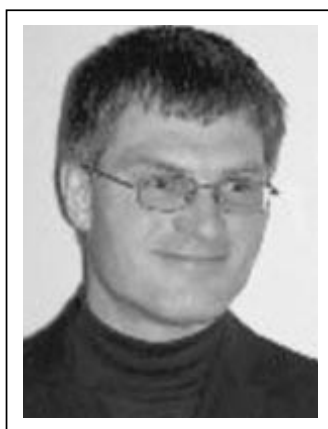

Prof. (FH) Mag. Dr. rer.soc.oec. Rainer Loidl-Keil, Jahrgang 1966, derzeit Studiengang Sozialarbeit und Sozialmanagement FH Joanneum Graz. Studium der Soziologie, Lehr- und Forschungsaufenthalte in Deutschland, USA, Irland, Polen. Vorträge und Publikationen im In- und Ausland. Schwerpunkte: Organisationen, Arbeit, Beruf, Wirtschaft,

Unternehmertum, Management, Führung; soziale Unternehmen und soziale Dienste, Sozialwirtschaft; Arbeitsmarkt, Qualifikationen; Forschungsmethoden, Evaluationsforschung.

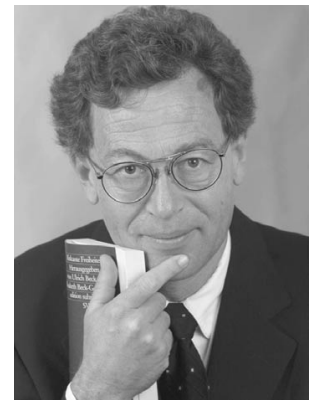

FH-Prof. HR. Mag. Dr. Klaus Posch ist seit 2001 Leiter des Studiengangs Sozialarbeit mit Ausbildungsschwerpunkt Sozialmanagement an der $\mathrm{FH} \mathrm{Jo-}$ anneum in Graz; zuvor war er Bewährungshelfer und leitete von 1983 bis 2001 die Bewährungshilfe Steiermark. Er studierte Evangelische Theologie, Psychologie und Soziologie an den Universitäten Wien und Salzburg und absolvierte außeruniversitäre Ausbildungen zum Psychoanalytiker und Gruppenpsychotherapeut. Er verfasste zahlreiche Publikationen u. a. auf den Gebieten der Sozialen Arbeit, insbesondere Methoden in der Sozialarbeit, klinische Psychologie (Dissozialität), Psychoanalyse und Sozialmanagement. Zurzeit ist er Leiter des Arbeitskreises für Psychoanalyse Linz/Graz, Vorsitzender des Steiermärkischen Jugendwohlfahrtsbeirats und Vorsitzender des Vereins Steirische Wohnplattform.

Standortgebundenheit und Individualität (Merchel 2006: $45,46)$. Doch fehlt die Präzisierung, was das spezifisch »Soziale« im Gegensatz zu anderen personenbezogenen Dienstleistungen ausmacht, für welche die Kennzeichen in gleicher Weise gelten. Zugleich wird der Geltungsbereich »sozialwirtschaftlicher« Leistungen über jene der "personenbezogenen sozialen Dienstleistungen « hinausgehen, weshalb die Charakterisierung für das beschriebene Feld zu stark verengt sein dürfte. 
Für eine Verortung der Sozialwirtschaft wählen wir eine alternative Vorgehensweise und nähern uns der begrifflichen Bestimmung ausgehend von der Annahme, dass die begriffliche Problematik substanziell mit dem dichotomen Begriffsmodell einhergeht. Die Begrifflichkeit eines »entweder Markt oder Staat « findet keine reale Entsprechung mehr. Sozialwirtschaft wird als eigener, klar einund abgrenzbarer und quasi »statischer « Bereich also schwerlich zu definieren sein - das entspräche nicht den Organisationswirklichkeiten.

Diese Umstände machen neue Wege der gesellschaftlichen als auch betrieblichen Positionierung der Sozialwirtschaft erforderlich. Um den realen Vielfältigkeiten der Sozialwirtschaft gerechter zu werden, schlagen wir deshalb eine begriffliche Modellierung vor, welche sozialwirtschaftlichen Organisationen deren qualitative Positionierung erlaubt und ihrer betrieblichen Realität adäquater wird. Eine mögliche Lösung präsentiert der Sozialwirtschaft-Kompass.

\section{Der Sozialwirtschaft-Kompass: zwei Achsen, eine Position}

Der Sozialwirtschaft-Kompass soll vorrangig den Unternehmen dienen, sich in einem heterogen strukturierten Feld selber zu verorten.

Die Verortung erfolgt hinsichtlich zweier zentraler organisatorischer Dimensionen oder im Kompass auf zwei Achsen: den Eigentumsverhältnissen und dem primären Unternehmenszweck. Auf der horizontalen Achse kann die Verortung zwischen öffentlicher Hand und Privatwirtschaft vorgenommen werden. Hier wird je nach Ownership (Besitz des Kapitals) des Unternehmens oder der Organisation eine Unterscheidung zwischen "Sozialverwaltung oder öffentliches Unternehmen“ und »Sozialwirtschaft oder Privatwirtschaft« vorgenommen. Die vertikale Achse spiegelt die Zielsetzung des Unternehmens oder der Einrichtung wider. Diese Dimension bewegt sich zwischen der sozialen Zielsetzung und der kommerziellen Zielsetzung als primären Unternehmenszweck (vgl. Abb. 1).

Selbstverortung und Eigendefinition werden der Fremddefinition vorgezogen, weil bei der Verwendung des Begriffes »sozial« im normativen Sinn oftmals individuelle Werte als Bezugsbasis herangezogen werden. Die Ergebnisse einer Einschätzung des Kompasses zeigen im Pilotprojekt, dass Fremdverortungen und Selbstpositionierungen nur gering voneinander abweichen.

\section{Die Eigentumsverhältnisse: formale Grenzziehung versus Erfahrungen in der Praxis}

Die Beweglichkeit zwischen den Polen »Public « und »Private« hängt von den Eigentumsverhältnissen oder vom »Ownership« ab. Diese klären auf, zu wie viel Prozent ein Unternehmen dem öffentlichen oder dem privaten Sektor zuzuordnen ist. Liegt der Besitz beispielsweise zu 100 Prozent in öffentlicher Hand, verortet sich das Unternehmen direkt auf der linken Grenzlinie. Auf einer handlungsorientierten Dimension sind weitere Aspekte

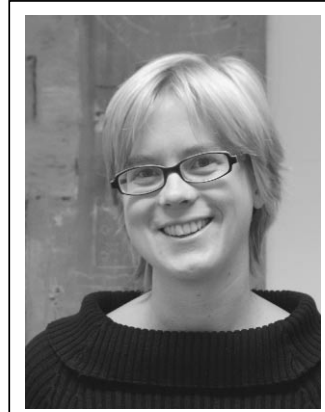

Mag.a Brigitte Kukovetz, geb. 1976, Graz, Soziologin, in der Entwicklung sozialer Leistungen für Menschen in benachteiligenden Situationen tätig. Tätigkeitsschwerpunkte: sozialwissenschaftliche und konzeptionelle Arbeiten zur Arbeitsmarktpolitik, Kooperation und Partnerschaften in der Sozialwirtschaft, Migration, Bildung, Geschlechterverhältnisse.

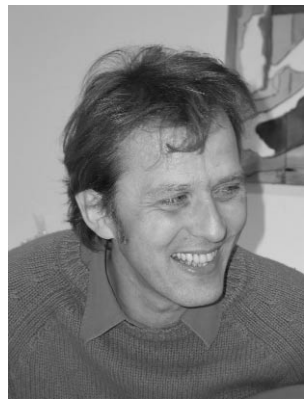

Ing. MMag. Manfred Leonhardt, geboren 1959 in Graz, Österreich, beruflicher Schwerpunkt ist Leadership in der Sozialwirtschaft durch Entwicklung und Vernetzung sozialer Leistungen.

des Unternehmertums angesprochen, die mit einbezogen werden, beispielsweise: Risikoverteilung, Entscheidungsfreiheit, Autonomie, Gestaltungsmöglichkeiten zur Reinvestition der Gewinne, Strukturierungsgrad und andere.

Da der Sozialwirtschaft-Kompass als Instrument zur Selbstverortung angelegt ist, ist eine flexible Anwendung denkbar. Es kann sowohl die Positionierung anhand der Eigentumsverhältnisse erfolgen, als auch im Sinne einer Reflexion anhand der weicheren Kriterien der handlungsorientierten Dimension.

\section{Der primäre Unternehmenszweck: Wann ist ein Unternehmen sozial?}

Auf der horizontalen Achse wird eine Positionierung hinsichtlich des primären Unternehmenszweckes vorgenommen. Bei einer kommerziellen Zielsetzung erfolgt die Verortung am unteren Limes, bei einer ausschließlich sozialen Zielsetzung am oberen. Die entscheidende Frage hierbei ist natürlich, wann von einer primären sozialen Zielsetzung gesprochen werden kann. Hierfür schlagen wir folgende Begriffsbestimmung vor. Der obere Limes ist erreicht, wenn ein Unternehmen oder eine Organisation folgende drei Kriterien erfüllt:

- In der Kerndefinition ist ein Unternehmen dann sozial, wenn dessen Handlungsmaximen auf moralischen Prinzipien basieren, die über einen ausgeprägten Egoismus hinausgehen (z. B. aufgeklärtes Eigeninteresse, Altruismus, moralischer Konventionalismus) basiert. Von anderen Autorinnen und Autoren wird dies als »ethische Fundierung « bezeichnet. Diese Kerndefinition entspricht einem Ideal, bei dessen Erfüllung die absolute Identität des Unternehmens gegeben ist. 
- Um eine Außenbeurteilung zur Orientierung, Zuordnung und Erkennung zu ermöglichen, kann definiert werden, dass ein Unternehmen als sozial dann gilt, wenn der primäre Unternehmens- oder Organisationszweck in der Verbesserung der Lebenssituationen und Lebenschancen benachteiligter Menschen und Menschengruppen und in der Wahrung gesellschaftlicher Schutz- und Erhaltungsinteressen liegt. Wann diese Menschen als sozial benachteiligt gelten, ist eine normative Frage und braucht einen Bezugspunkt: benachteiligt im Vergleich zu jemanden, benachteiligt in Bezug auf etwas. Benachteiligung kann nur unter Vorlage spezifischer moralischer Prinzipien einerseits und realer Lebenskontexte andererseits stattfinden. Dieser Bezugspunkt kann in einem demokratisch legitimierten Staat nur dessen Handlungsmaxime sein, in dessen Mittelpunkt das Gemeinwohl steht. Den Bürgerinnen und Bürgern des demokratisch legitimierten Rechtsstaates sollen gute Lebens- und Entfaltungschancen verfügbar sein. Was nun der Staat als Gemeinwohl ansieht, ist in dessen Gesetzgebung ersichtlich. In dieser Auslegung sind Verfassungen und (Städte-) Statuten geeignete Indikatoren für den »kleinsten gemeinsamen Nenner «, was in einer demokratisch organisierten Gesellschaft als Gemeinwohl angesehen wird. Darüber hinaus drückt die jeweils gewählte Regierung den Status quo über die Gemeinwohlvorstellungen der Gesellschaft aus; auf weiteren Ebenen finden wir Bezugspunkte in den Landesgesetzgebungen, möglicherweise noch auf Ebene der Regionen und Gemeinden, die für sich und die jeweilige Region Gemeinwohl spezifizieren können. Sind nun die durch das demokratische Rechtssystem legitimierten Grundsätze und Vorstellungen darüber, was in einer Gesellschaft Existenzabsicherung, Daseinsvorsorge und grundlegende Lebensbedingungen und gesellschaftliche Teilhabe bedeuten, nicht von allen Menschen oder Menschengruppen zu gleichen Teilen wahrnehmbar, ist von sozialer Benachteiligung zu sprechen. Wir betonen, dass in dieser Argumentation keine normative oder empfehlende Feststellung vorgenommen wird, »was « als soziale Benachteiligung gilt oder gelten soll. Es geht viel mehr um das Auffinden eines Bezugspunktes für eine mögliche Definition der im Alltagsgebrauch individuell und wertend verwendeten Begriffe »sozial « und »Benachteiligung «.

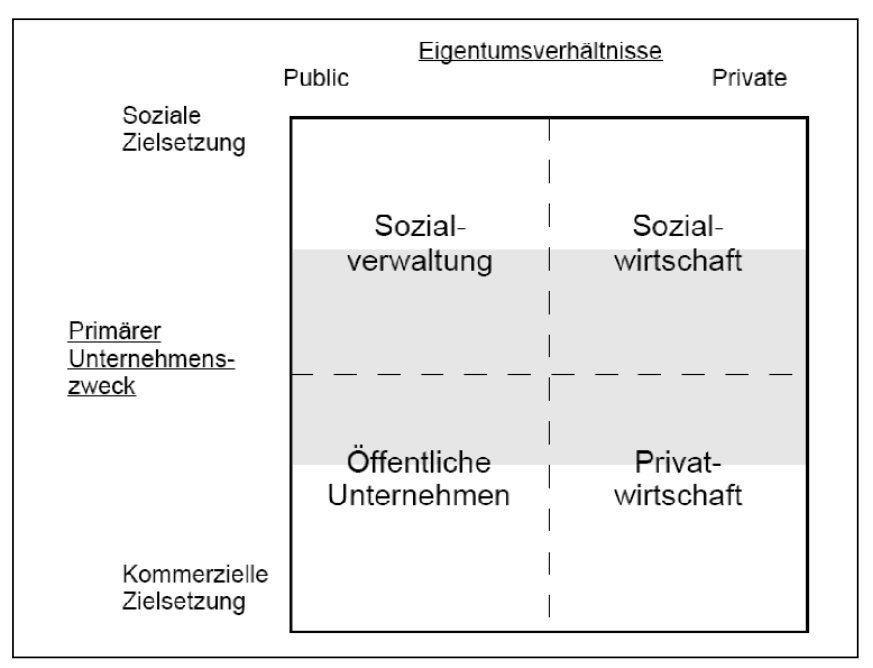

Abb. 1: Mit dem Sozialwirtschafts-Kompass können mittels vier Dimensionen Unternehmen und Organisationen verortet werden.
- Das dritte und das für eine qualitative Identifizierung am besten geeignete Kriterium stellt jenes der sozialökonomischen Wertschöpfung und Gewinnverwendung dar. Sowohl der erwirtschaftete Gewinn als auch die Ressourcen werden im Sinne der sozialen Unternehmensmission nachweislich für die benachteiligten Menschen und Menschengruppen reinvestiert.

Nicht als definitorisches Kriterium, sondern als beschreibendes Merkmal im Sinne einer oftmaligen Funktion der Sozialwirtschaft kann eine weitere Dimension herangezogen werden, jene der »Intermediarität «: Sozialwirtschaftliche Unternehmen und Organisationen sind sich ihrer intermediären Funktion zwischen öffentlichen Organisationen und privatwirtschaftlichen Unternehmen bewusst und können diese, in Bezug setzend zu obigen Kriterien, darstellen und ausüben.

Sind die genannten Kriterien vollständig erfüllt, positioniert sich ein Unternehmen am obersten Limes des Sozialwirtschaft-Kompasses. Sind nur Teile davon für ein Unternehmen gültig, wird das Unternehmen oder die Organisation zwischen den beiden horizontalen Limes oben und unten verortet. Dies kann beispielsweise der Fall sein, wenn ein Unternehmen oder eine Organisation soziale Zielsetzungen zwar nicht als primären Unternehmenszweck, jedoch in großen Bereichen und zu großen Anteilen verfolgt.

Ist jedoch keines der oben genannten Kriterien erfüllt oder liegt der primäre Unternehmenszweck in der Verfolgung einer kommerziellen Zielsetzung, dann ist das Unternehmen oder die Organisation an der unteren horizontalen Linie zu verorten.

\section{Ergebnisse aus der Anwendung und Konklusion}

Der hier präsentierte Sozialwirtschaft-Kompass wurde in der österreichischen Entwicklungspartnerschaft »Public Social Private Partnership« entwickelt und erprobt (vgl. Abb. 2). Die Einordnung der Organisationen und Unternehmen erfolgt von den jeweiligen Geschäftsführungen oder Mitarbeiterschaft selbst. Es wird ein Mittelwert errechnet, falls mehrere Mitarbeiterinnen und Mitarbeiter eine Verortung vornehmen. Als Ergebnis dieser beispielhaften Anwendung kann festgehalten werden:

- Das Instrument wird von den befragten Personen gut angenommen.

- Die Selbstverortungen in die vier vordefinierten Zellen ermöglicht eine angemessene Unterscheidung der verschiedenen gesellschaftlichen Felder (sowohl zwischen Sozialwirtschaft und Privatwirtschaft, aber auch zwischen öffentlich und privat).

Zusätzlich wird ein Vergleich der Selbsteinschätzungen mit Fremdeinschätzungen vorgenommen. Anhand von fünf Unternehmen wird erkundet, inwieweit die eigene Positionierung übereinstimmt damit, wie außenstehende Personen das Unternehmen oder die Organisation verorten würden. Hierzu werden mehrere Personen befragt, mehrere Mitarbeiterinnen und Mitarbeiter einer Organisation und mehrere Personen, die die jeweilige Organisa- 


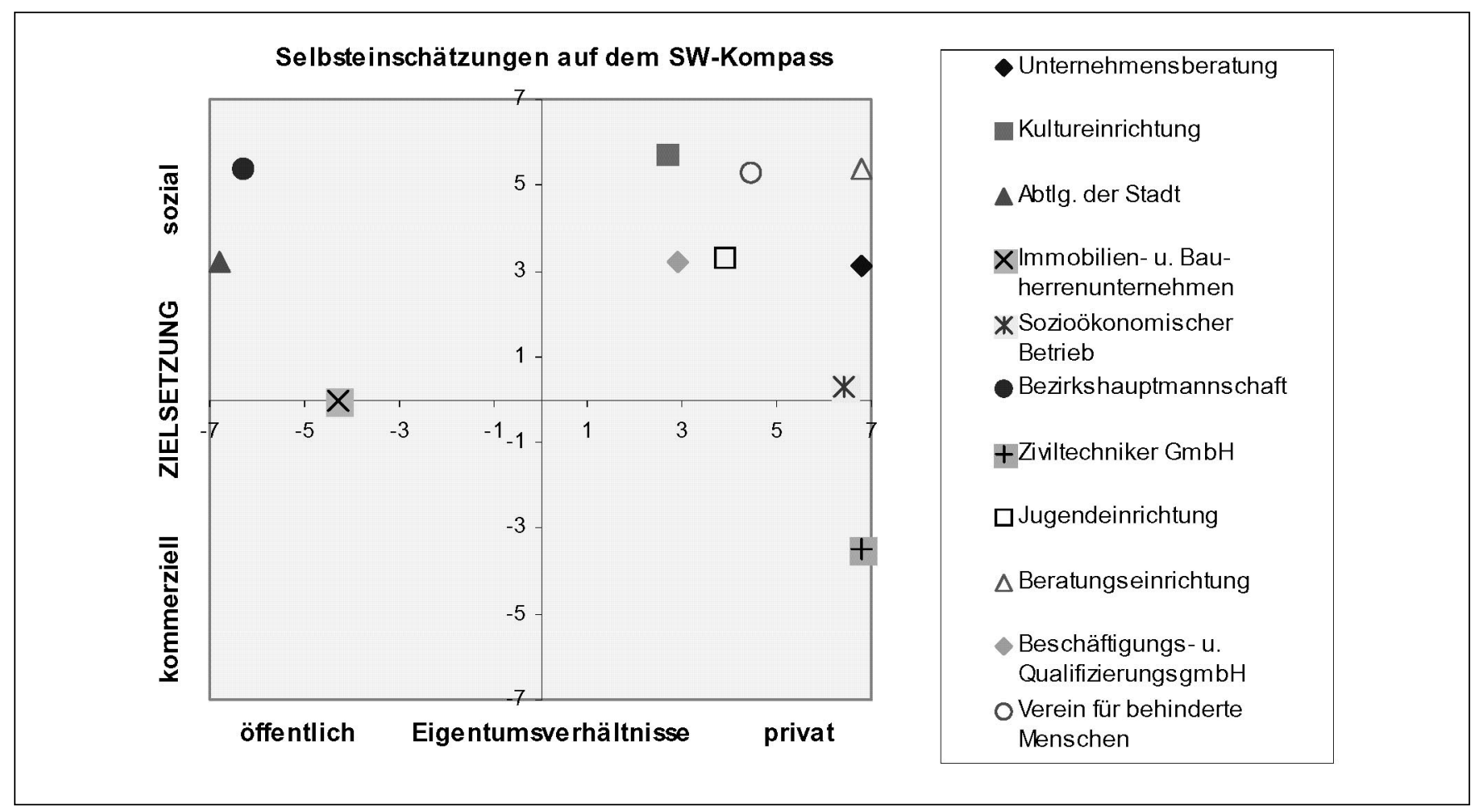

Abb. 2: Mit verschiedenen Beispielen kann die praktische Anwendung des Sozialwirtschafts-Kompasses demonstriert werden.

tion von außen verorteten. Werden alle Verortungen miteinbezogen, sind beträchtliche Unterschiede zwischen selbst- und fremdbestimmter Positionierung feststellbar. Diese Ergebnisse zeigen, dass die einzelnen Personen sehr unterschiedliche Einschätzungen abgeben, was die oftmals sehr kontroverse Diskussion um die Definition und Abgrenzung der Sozialwirtschaft widerspiegelt. onsbild herstellen. Zum einen befördert eine weniger vage Positionierung die deutlichere Identifizierung und Kollektivierung individueller Zielsetzungen innerhalb der Organisationsmitglieder, zum anderen werden Zielsetzungen exakter formuliert und Orientierungen können in den Bezügen der Organisation zu ihrem Milieu besser vermittelt werden.

\section{"Innovationen lassen sich nicht administrativ verordnen, sondern brauchen unternehmerische Autonomie«}

Die Mittelwerte der unterschiedlichen individuellen Einschätzungen hingegen zeigen auch, dass die durchschnittlichen Fremdverortungen mit den durchschnittlichen Selbstverortungen korrespondieren. Daraus lässt sich schließen, dass der Sozialwirtschaft-Kompass nicht nur aufgrund der eigenen selbstreflexiven Positionierungen, sondern auch auf Basis von Fremdverortungen eine angemessene Unterscheidung der verschiedenen gesellschaftlichen Felder und der Verortung der Unternehmen und Organisationen darin ermöglicht.

\section{Nutzen für Unternehmenssteuerung, Positionierung und Ordnungspolitik}

Der Sozialwirtschaft-Kompass kann Organisationsgestaltung und Unternehmenssteuerung bereichern, denn eine jede Organisation muss für Identitätsbildung und Sinnstiftung nach innen und außen ein kollektives Organisati-
Steuerung sozialwirtschaftlicher Unternehmen bedeutet zudem, Eigentumsverhältnisse und Auftragsverhältnisse in einer komplexen Stakeholder-Struktur zu kennen und sich der Abhängigkeiten und wechselseitigen Bedingtheiten bewusst zu sein. So müssen Organisationen auch ihre Außenbeziehungen gestalten, sich in ihrem Umfeld und im permanenten Austausch bewähren, ihren Bestand zwischen Kooperation und Konkurrenz sichern. Für Klärungen dieser Art, wie also Kontexte auf eine Organisation wirken und wie eine Organisation auf ihren Kontext wirken kann, und das Einnehmen und ein bewussteres Gestalten solcher Organisationsbeziehungen kann eine Matrix wie der Sozialwirtschaft-Kompass vereinheitlichend und verständnisbildend wirken. In Folge dürften beispielsweise Interessenlagen besser ersichtlich und aushandelbar werden.

Gerade in einem Kontext wie jenem sozialwirtschaftlicher Unternehmen ist die Frage nach der Verfügbarkeit von Ressourcen ständig virulent. Dass der Leistungsprozess von Ressourcen abhängig ist, dass die Organisation zur Bestandssicherung »Inputs « benötigt und für die Umwelt akzeptable »Outputs « erstellt, ist offenkundig und gilt für alle Unternehmen. Das Ressourcen-Abhängigkeits-Theorem verweist uns darauf, dass künftige Ressourcen ungewiss sind, und dass sowohl die Effizienz des Leistungspro- 
zesses als auch die Planung von Aktivitäten latent bedroht sind. In noch höherem Maße als in kommerziellen mag angesichts der strukturellen Ressourcenkomplexität für sozialwirtschaftliche Unternehmen gelten, dass sie klären müssen, von welchen Ressourcen die Organisation abhängig ist; welche Handlungsstrategien die Organisation entwickeln kann, um an Ressourcen zu kommen oder um mit latenter Unsicherheit umzugehen. Der SozialwirtschaftKompass leistet hierbei keinesfalls die Strategiebildung an sich, doch ermöglichen die Positionsbestimmungen potenziell klarere Ausgangssituationen zur Strategiebildung, um Unsicherheiten und Abhängigkeiten besser zu erkennen und schließlich zu reduzieren.

Sozialwirtschaftliche Unternehmen finden sich häufig in Situationen zwischen »ökonomischer Diskreditierung « und »administrativen Erfüllungsgehilfen der öffentlichen Hand « wieder. Mit dem Sozialwirtschaft-Kompass wird demgegenüber eine Haltung befördert, die von der Eigenständigkeit sozialwirtschaftlicher Unternehmen ausgeht, und davon, dass es sich hierin um spezifische Wertschöpfungsprozesse handelt. Innovationen lassen sich kaum »administrativ« lösen, sondern bedürfen der Anerkennung der Bedeutung unternehmerischer Autonomie.

Sozialwirtschaftliche Unternehmen sehen sich zusehends Komplexitätszuwächsen und neuen Akzeptabilitätsund Legitimitätsgrundlagen gegenüber - Ansprüche steigen nicht nur vor dem Hintergrund der Transformationen in Verwaltung und Ökonomie. Soziale Dienstleistungen können von sozialwirtschaftlichen ebenso wie von privatwirtschaftlichen als auch öffentlichen Organisationen erbracht werden, was zugleich die Frage nach der Spezifität sozialwirtschaftlicher Unternehmen aufwirft.

Die Matrix des Sozialwirtschaft-Kompasses beantwortet diese in zwei Richtungen, als die spezifische Unternehmensmission und Unternehmensfunktion normative und strukturelle Implikationen birgt und diese miteinander verknüpft werden. Zum einen geht es um die Auslegung von Profit (Organisationsziel), worin sich der Typus sozialwirtschaftlicher Unternehmen als eine Organisationsform neben anderen in der Weise positioniert: Der Typus steht für die explizite Wahrung und Vorrangigkeit sozialer Organisationsziele und Organisationsinhalte. Zum anderen wird ein arbeitsorganisatorischer Aspekt angesprochen, die betriebswirtschaftliche Leistungserbringung, die Verfügbarkeit von Betriebsmittel. In diesem Aspekt von Eigentumsverhältnissen und Auftragsverhältnissen geht es in einem weiteren Verständnis insbesondere um Möglichkeiten der Einflussnahme und um die Verteilung von Steuerung, Macht, Kontrolle, Partizipation; um die Frage, in welchem Organisationstyp in welcher Weise für wen die Teilhabe an Gestaltungsprozessen expliziert ist.

\section{Resümee}

Der Sozialwirtschaft-Kompass ermöglicht das Einnehmen einer exklusiven Position, für die in der traditionsbehafteten Perspektive von »Markt und Staat « kein Formtypus vorgesehen ist: in Form der sozialwirtschaftlichen Unternehmen werden Leistungserstellung und Wertschöpfungsprozess idealiter unabhängiger sowohl von parteipolitischen als auch ökonomischen Einzelinteressen.
Aus klassischer sozial- und ordnungspolitischer Sicht kennen wir zwei Institutionen: Markt und Staat. Die Emergenz einer dritten Institution wird im Teilversagen anderer Institutionen begründet. Als »komplementäre Dritte« bleibt sie letzten Endes positionslos und nur definierbar aus Existenz und Versagen anderer. Der Sozialwirtschaft-Kompass beschreitet einen anderen Weg. Mit dem Einführen zweier grundlegender konstitutioneller Dimensionen sind strukturfunktional vier Felder für verschiedene Kombinationen von Organisationszielen und Eigentumsverhältnissen vorgesehen. Der Sozialwirtschaft-Kompass als Matrix ordnet funktional erforderliche Erscheinungsformen neu entlang zweier Achsen. Die aufgebürdeten Fragen nach Nützlichkeit, Notwendigkeit oder Legitimität der Form »Sozialwirtschaft « stellen sich so nicht mehr. Die Sozialwirtschaft nimmt wie die drei anderen Formtypen eine bestimmte Position ein. Gibt es eine der vier Formen nicht, bleibt ein Feld leer, wird ein Funktionsfeld nicht erfüllt.

Im Vergleich zu herkömmlichen begrifflichen Bestimmungen ermöglicht der Sozialwirtschaft-Kompass eine alternative Perspektive und eine praxisorientierte und dialogische Verortung. Üblicherweise werden Definitionen entworfen, Inklusions- und Exklusions-Kriterien entwickelt und Merkmale expliziert, die dann in der praktischen Verortung nicht einhaltbar sind. Der Sozialwirtschaft-Kompass erlaubt hingegen eine der Vielfältigkeit der Praxis entsprechende »weichere « Verortung. Damit sind eine für die Praxis möglicherweise angemessenere Navigation und Identitätsfindung sowie bessere Verständigung zwischen Selbstbestimmung und Fremdbestimmung gegeben.

Der voranstehende Artikel resultiert aus der Entwicklungspartnerschaft »Public Social Private Partnership « in Österreich. Diese wird im Rahmen der Gemeinschaftsinitiative EQUAL aus Mitteln des Europäischen Sozialfonds und aus Mitteln des Wiener Bundesministeriums für Wirtschaft und Arbeit gefördert. Neben den Autoren dieses Beitrags haben den Sozialwirtschaft-Kompass mitentwickelt: Martina Haas, Andreas Thomasser und Peter Wilhelmer.

Kontakte: rainer.loidlkeil@fh-joanneum.at und info@sat3.at. 